\title{
The Banach space $S$ is complementably minimal and subsequentially prime
}

\author{
by \\ G. Androulakis (Columbia, SC) and \\ T. Schlumprecht (College Station, TX)
}

\begin{abstract}
We first include a result of the second author showing that the Banach space $S$ is complementably minimal. We then show that every block sequence of the unit vector basis of $S$ has a subsequence which spans a space isomorphic to its square. By the Pełczyński decomposition method it follows that every basic sequence in $S$ which spans a space complemented in $S$ has a subsequence which spans a space isomorphic to $S$ (i.e. $S$ is a subsequentially prime space).
\end{abstract}

1. Introduction. The Banach space $S$ was introduced by the second author as an example of an arbitrarily distortable Banach space [14]. In [8] the space $S$ was used to construct a Banach space which does not contain any unconditional basic sequence. In this paper we are concerned with the question whether or not $S$ is a prime space. We present two partial results: In Section 2 we include a result proved by the second author some time ago but not published until now [15], that $S$ is complementably minimal, and thereby answer a question of P. G. Casazza, who asked whether or not $\ell_{p}, 1 \leq p \leq \infty$, and $c_{0}$ are the only complementably minimal spaces. In Section 3 we prove that $S$ is subsequentially prime.

Let us recall the above notions. A Banach space $X$ is called prime [12] if every complemented infinite-dimensional subspace of $X$ is isomorphic to $X$. A. Pełczyński [13] showed that the spaces $c_{0}$ and $\ell_{p}(1 \leq p<\infty)$ are prime, and J. Lindenstrauss [11] showed that this is also true for the space $\ell_{\infty}$. New prime spaces were constructed by W. T. Gowers and B. Maurey [9]. But it is still open whether or not $\ell_{p}, 1 \leq p \leq \infty$, and $c_{0}$ are the only prime spaces with an unconditional basis.

A space $X$ is called minimal (a notion due to $H$. Rosenthal) if every infinite-dimensional subspace of $X$ contains a subspace isomorphic to $X$, and $X$ is called complementably minimal [5] if every infinite-dimensional

2000 Mathematics Subject Classification: 46B03, 46B20.

Research of both authors supported by NSF. 
subspace of $X$ contains a subspace which is isomorphic to $X$ and complemented in $X$. P. G. Casazza and E. Odell [4] showed that Tsirelson's space $T$ (see [16]), as described in [6], fails to have a minimal subspace. On the other hand it was shown by P. G. Casazza, W. B. Johnson and L. Tzafriri [3] that the space $T^{*}$ is minimal but not complementably minimal. Since $S$ is complementably minimal, either $S$ is prime, or there exists a complemented subspace $X$ of $S$ such that $X$ and $S$ give a negative solution to the Schroeder-Bernstein problem for Banach spaces (see [2] for a detailed discussion of this question): if two spaces are isomorphic to complemented subspaces of each other must they be isomorphic? Negative solutions to the Schroeder-Bernstein problem for Banach spaces are given by W. T. Gowers [7], and W. T. Gowers and B. Maurey [9], but to our knowledge it is open whether or not there are two Banach spaces $X$ and $Y$, both having an unconditional basis, so that $X$ is complemented in $Y$ and $Y$ is complemented in $X$, but so that $X$ and $Y$ are not isomorphic.

The following terminology was suggested to us by D. Kutzarova.

Definition. A Banach space $X$ with a basis is called subsequentially prime if for every basic sequence $\left(x_{i}\right)$ of $X$ such that the closed linear span of $\left(x_{i}\right)$ is complemented in $X$, there exists a subsequence $\left(y_{i}\right)$ such that the closed linear span of $\left(y_{i}\right)$ is isomorphic to $X$.

As mentioned above, we will show that the space $S$ is subsequentially prime. We do not know if $S$ is prime, we even do not know whether or not the closed linear span of a block basis which is complemented in $S$ is isomorphic to $S$.

We will need some notations. Let $c_{00}$ be the linear span of finitely supported real sequences, and let $\left(e_{i}\right)$ denote its standard basis. For $x \in c_{00}$, $\operatorname{supp}(x)=\left\{i \in \mathbb{N}: x_{i} \neq 0\right\}$ denotes the support of $x$. For a finite set $A$ the cardinality of $A$ is denoted by $\# A$. If $E, F \subset \mathbb{N}$ we write $E<F$ if $\max E<\min F$, and we write $x<y$ for $x, y \in c_{00}$ if $\operatorname{supp}(x)<\operatorname{supp}(y)$. A sequence $\left(x_{i}\right)_{i}$ in $c_{00}$ is a block sequence of $\left(e_{i}\right)$ if $x_{1}<x_{2}<\ldots$ For $x=\sum_{i \in \mathbb{N}} x_{i} e_{i} \in c_{00}$ and $E \subset \mathbb{N}, E(x)$ is the projection of $x$ onto the span of $\left(e_{i}\right)_{i \in E}$, i.e.

$$
E(x)=\sum_{i \in E} x_{i} e_{i}
$$

Recall [14] that the norm of $S$ is the unique norm on the completion of $c_{00}$ which satisfies the implicit equation

$$
\|x\|=\|x\|_{\ell_{\infty}} \vee \sup _{\substack{2 \leq n, E_{i} \subseteq \mathbb{N}, i=1, \ldots, n \\ E_{1}<\ldots<E_{n}}} \frac{1}{f(n)} \sum_{i=1}^{n}\left\|E_{i}(x)\right\|
$$

where $\|\cdot\|_{\ell_{\infty}}$ denotes the norm of $\ell_{\infty}$ and $f(n)=\log _{2}(n+1)$ for $n \in \mathbb{N}$. For 
$x \in S$ and $l \in \mathbb{N}, l \geq 2$, we define

$$
\|x\|_{l}:=\sup _{E_{1}<\ldots<E_{l}} \frac{1}{f(l)} \sum_{i=1}^{l}\left\|E_{i}(x)\right\| .
$$

We note that $\|\cdot\|_{l}, 2 \leq l<\infty$, is an equivalent norm on $S$ and we observe that for $x \in S$ and $2 \leq l<\infty$ we have

$$
\frac{1}{f(l)}\|x\| \leq\|x\|_{l} \leq\|x\| \quad \text { and } \quad\|x\|=\sup _{2 \leq l \leq \infty}\|x\|_{l} .
$$

Finally for any $2 \leq r<\infty$ and $x \in S$ we put

$$
\|x\|_{r}:=\sup _{\substack{l \geq r \\ l \in \mathbb{N} \cup\{\infty\}}}\|x\|_{l} .
$$

Two sequences $\left(x_{i}\right),\left(y_{i}\right)$ in $S$ are called $c$-equivalent, for some $c \geq 1$, and we write $\left(x_{i}\right) \approx_{c}\left(y_{i}\right)$, if $\left\|\sum a_{i} x_{i}\right\| \stackrel{c}{\approx}\left\|\sum a_{i} y_{i}\right\|$ for all $\left(a_{i}\right) \in c_{00}$, where for $c \geq 1$ and $a, b \geq 0$ we write $a \stackrel{c}{\approx} b$ to denote that $(1 / c) a \leq b \leq c a$. If $\left(x_{i}\right)$ and $\left(y_{i}\right)$ are $c$-equivalent for some $c>1$ we write $\left(x_{i}\right) \approx\left(y_{i}\right)$. A basic sequence $\left(x_{n}\right)$ is called $c$-subsymmetric if it is $c$-unconditional and $c$-equivalent to all of its subsequences. For two Banach spaces $X$ and $Y$ we write $X \approx_{c} Y$ if there is an isomorphism $T$ between $X$ and $Y$ with $\|T\| \cdot\left\|T^{-1}\right\| \leq c$, and we write $X \approx Y$ if $X \approx_{c} Y$ for some $c \geq 1$.

If $\left(x_{n}\right)$ is a sequence in a Banach space then $\left[x_{n}: n \in \mathbb{N}\right]$ denotes the closed linear span of $\left(x_{n}\right)$. If not said otherwise, all statements in the following sections refer to the space $S$.

We would like to thank P. G. Casazza and D. Kutzarova for valuable discussions.

2. The Banach space $S$ is complementably minimal. The goal of this section is the proof of the following theorem.

THEOREM 2.1. S is complementably minimal.

First recall the following result which follows from Lemma 5 of [14].

Proposition 2.2. $\ell_{1}$ is block finitely represented in each block basis of $\left(e_{i}\right)$, i.e., if $\varepsilon>0$ and $m \in \mathbb{N}$, and if $\left(y_{n}\right)$ is a block basis of $\left(e_{i}\right)$, then there is a block basis $\left(z_{i}\right)_{i=1}^{m}$ of $\left(y_{n}\right)$ which is $(1+\varepsilon)$-equivalent to the unit basis of $\ell_{1}^{m}$, i.e.,

$$
\left\|\sum_{i=1}^{m} \alpha_{i} z_{i}\right\| \geq \frac{1}{1+\varepsilon} \sum_{i=1}^{m}\left|\alpha_{i}\right|
$$

for $\left(\alpha_{i}\right)_{i=1}^{m} \subset \mathbb{R}$. 
The proof of the following statement can be compiled from the proof of Theorem 3 of [14]. Since the statement is crucial for our main result we include its proof.

Lemma 2.3. Let $\varepsilon>0$ and $l \in \mathbb{N}$. Then there is an $n=n(\varepsilon, l) \in \mathbb{N}$ with the following property: If $m \geq n$ and if $y=m^{-1} \sum_{i=1}^{m} x_{i}$, where $\left(x_{i}\right)_{i=1}^{m}$ is a normalized block basis of $\left(e_{i}\right)$ which is $(1+\varepsilon / 2)$-equivalent to the unit basis of $\ell_{1}^{m}$, then

$$
\sup _{E_{1}<\ldots<E_{l}} \sum_{i=1}^{l}\left\|E_{i}(y)\right\| \leq\|y\|+\varepsilon \leq 1+\varepsilon .
$$

Proof. Let $n \in \mathbb{N}$ so that $4 l / n \leq \varepsilon$ and assume $m \geq n$ and $\left(x_{i}\right)_{i=1}^{m}$ are as in the statement. Furthermore, let $E_{1}<\ldots<E_{l}$ be finite subsets of $\mathbb{N}$. Since $\left(e_{i}\right)$ is 1-unconditional we can assume that the $E_{j}$ 's are intervals in $\mathbb{N}$. This implies that for each $j \in\{1, \ldots, l\}$ there are at most two elements $i_{1}, i_{2} \in\{1, \ldots, m\}$ so that $E_{j} \cap \operatorname{supp}\left(x_{i_{s}}\right) \neq \emptyset$ and $\operatorname{supp}\left(x_{i_{s}}\right) \backslash E_{j} \neq \emptyset$, $s=1,2$. For $j=1, \ldots, l$, let

$$
\widetilde{E}_{j}=\bigcup\left\{\operatorname{supp}\left(x_{i}\right): i \leq m \text { and } \operatorname{supp}\left(x_{i}\right) \subset E_{j}\right\} .
$$

It follows that if $y=m^{-1} \sum_{i=1}^{m} x_{i}$, then $\left\|E_{i}(y)-\widetilde{E}_{i}(y)\right\| \leq 2 / m$ and from the assumption that $\left(x_{i}\right)_{i=1}^{m}$ is $(1+\varepsilon / 2)$-equivalent to the $\ell_{1}^{m}$ unit basis we deduce that

$$
\begin{aligned}
\sum_{j=1}^{l}\left\|E_{j}(y)\right\| & \leq \frac{2 l}{m}+\sum_{j=1}^{l}\left\|\widetilde{E}_{j}(y)\right\|=\frac{2 l}{m}+\frac{1}{m} \sum_{j=1}^{l}\left\|\sum_{\operatorname{supp}\left(x_{i}\right) \subset \widetilde{E}_{j}} x_{i}\right\| \\
& \leq \frac{2 l}{m}+\frac{1}{m}\left\|\sum_{i=1}^{m} x_{i}\right\|(1+\varepsilon / 2) \leq \varepsilon+\|y\| \leq \varepsilon+1 .
\end{aligned}
$$

The following theorem essentially proves that $S$ is minimal. We postpone its proof.

TheOREM 2.4. Let $\left(\varepsilon_{n}\right) \subset \mathbb{R}^{+}$with $\sum \varepsilon_{n}<\infty$ and let $\left(y_{n}\right)$ be a normalized block basis of $\left(e_{n}\right)$ with the following properties: There is a sequence $k_{n} \uparrow \infty$ in $\mathbb{N}$ so that for all $n \in \mathbb{N}$,

$$
\begin{aligned}
& \sup _{\substack{k \leq k_{n-1} \\
E_{1}<\ldots<E_{k}}} \sum_{i=1}^{k}\left\|E_{i}\left(y_{n}\right)\right\| \leq 1+\varepsilon_{n}, \\
& \max \operatorname{supp}\left(y_{n}\right) \leq \varepsilon_{n} f\left(k_{n} / 3\right) .
\end{aligned}
$$

Then $\left(y_{n}\right)$ is equivalent to $\left(e_{n}\right)$.

Proof of Theorem 2.1. By the usual perturbation argument we only have to show that every block basis $\left(z_{n}\right)$ of $\left(e_{n}\right)$ has a further block basis which is equivalent to $\left(e_{n}\right)$. Letting for example $\varepsilon_{i}=2^{-i}, i=1,2, \ldots$, we have to 
find a normalized block $\left(y_{n}\right)$ of $\left(z_{n}\right)$ and a sequence $\left(k_{n}\right)$ in $\mathbb{N}$ so that (2) and (3) of Theorem 2.4 are satisfied. Indeed, put $k_{0}=1$ and assume that $k_{0}<k_{1}<\ldots<k_{n}$ and $y_{1}<\ldots<y_{n}$ are already defined for some $n \geq 0$. By Remark 2.2 and Lemma 2.3 we can choose $y_{n+1}>y_{n}$ in the linear span of $\left(z_{i}\right)$ so that condition (2) of Theorem 2.4 is satisfied. Since $\lim _{i \rightarrow \infty} f(i)=\infty$ we can then choose $k_{n+1}$ so that (3) is true.

In order to show that $S$ is complementably minimal we first observe that (1) implies that every normalized block basis $\left(y_{n}\right)$ of $\left(e_{n}\right)$ dominates $\left(e_{n}\right)$, i.e., that $\left\|\sum_{i=1}^{\infty} \alpha_{i} y_{i}\right\| \geq\left\|\sum_{i=1}^{\infty} \alpha_{i} e_{i}\right\|$ for all $\left(\alpha_{i}\right) \in c_{00}$. Secondly, we apply the following more general proposition which finishes the proof of Theorem 2.1.

Proposition 2.5. Let $Z$ be a Banach space with a $c_{u}$-unconditional basis $\left(e_{n}\right), c_{u} \geq 1$. Assume furthermore that there is a $c_{d}>0$ so that every normalized block basis $\left(y_{n}\right)$ of $\left(e_{n}\right) c_{d}$-dominates $\left(e_{n}\right)$ (i.e., $c_{d}\left\|\sum \alpha_{i} y_{i}\right\| \geq$ $\left\|\sum \alpha_{i} e_{i}\right\|$ for all $\left.\left(\alpha_{i}\right) \in c_{00}\right)$. Then a subspace of $Z$ generated by a normalized block of $\left(e_{n}\right)$ which is equivalent to $\left(e_{n}\right)$ is complemented in $Z$.

Proof. Without loss of generality we can assume that $\left(e_{n}\right)$ is a normalized and bimonotone basis of $Z$ (i.e. $\|[m, n](z)\| \leq\|z\|$ for all $z=\sum_{i=1}^{\infty} z_{i} e_{i} \in Z$, and $1 \leq m \leq n$ in $\mathbb{N})$. Assume that $\left(y_{n}\right)$ is a block of $\left(e_{i}\right)$ which is $c_{e^{-}}$ equivalent to $\left(e_{i}\right)$. Using the assumption that $\left(e_{n}\right)$ is normalized and bimonotone we find $y_{n}^{*} \in Z^{*}$, for $n \in \mathbb{N}$, with $1=\left\|y_{n}^{*}\right\|=y_{n}^{*}\left(y_{n}\right)$ and $\operatorname{supp}\left(y_{n}^{*}\right) \subset$ $\left[1+\max \operatorname{supp}\left(y_{n-1}\right), \max \operatorname{supp}\left(y_{n}\right)\right]\left(\right.$ where $y_{0}=0$ and $\left.\max (\emptyset)=0\right)$. Define $T=\sum y_{n}^{*} \otimes y_{n}, x \mapsto \sum y_{n} y_{n}^{*}(x)$. We have to show that $T$ is well defined and bounded on $Z$; then it easily follows that it is a projection on $\left[y_{n}: n \in \mathbb{N}\right]$. Let $x=\sum a_{i} e_{i}$ with $\left(a_{i}\right) \in c_{00}$. We can write $x=\sum x_{i}=\sum\left\|x_{i}\right\| u_{i}$ with $x_{i}=\left[1+\max \operatorname{supp}\left(y_{i-1}\right), \max \operatorname{supp}\left(y_{i}\right)\right](x)$ and $u_{i}=x_{i} /\left\|x_{i}\right\|$ if $x_{i} \neq 0$, and $u_{i}=e_{\max \operatorname{supp}\left(y_{i}\right)}$ otherwise. Then it follows that

$$
\begin{aligned}
T(x) & =\left\|\sum y_{n} y_{n}^{*}\left(x_{n}\right)\right\| \leq c_{u}\left\|\sum\right\| x_{n}\left\|y_{n}\right\| \leq c_{u} c_{e}\left\|\sum\right\| x_{n}\left\|e_{n}\right\| \\
& \leq c_{u} c_{e} c_{d}\left\|\sum\right\| x_{n}\left\|u_{n}\right\|=c_{u} c_{e} c_{d}\|x\| .
\end{aligned}
$$

Let $x \in S$. If $l$ is the smallest element of $\mathbb{N}$ so that $\|x\|=\|x\|_{l}$, we call $l$ the character of $x$ and write $\operatorname{char}(x)=l$. If $\|x\|=\|x\|_{\ell_{\infty}}$ we write $\operatorname{char}(x)=\infty$.

The next lemma makes the following qualitative statement precise: If $x \in c_{00}$, if $r>1$ is "big enough", and if $E_{1}<\ldots<E_{l}, l \geq r$, are subsets of $\mathbb{N}$ so that

$$
\|x\|_{r}=\frac{1}{f(l)} \sum_{i=1}^{l}\left\|E_{i}(x)\right\|,
$$

then for "most of the $E_{i}$ 's" the character of $E_{i}(x)$ is "much bigger than $r$ ". 
Lemma 2.6. There is a constant $d>1$ so that for all $r \in \mathbb{R}_{+}$with $f(r)>d^{2}$,

$$
\|x\|_{r} \leq\left[\frac{1}{1-d / \sqrt{f(r)}}\right] \sup _{\substack{l \geq r \\ E_{1}<\ldots<E_{l}}} \frac{1}{f(l)} \sum_{i=1}^{l}\left\|E_{i}(x)\right\|_{r} f(r)
$$

if $x \in c_{00}$ with $\|x\|_{r} \neq\|x\|_{\ell_{\infty}}$.

Proof. From the logarithmic behavior of $f$ we deduce that there is a constant $c>2$ so that the following inequalities hold:

$$
\begin{aligned}
f(\xi)-1 & \geq f(\xi) / c & & \text { whenever } \xi \geq 2, \\
c f(\xi) & \geq f\left(\xi \xi^{\prime}\right)-f(\xi) & & \text { whenever } \xi, \xi^{\prime} \geq c, \\
f\left(\xi^{1 / \sqrt{f(\xi)}}\right) & \leq c \sqrt{f(\xi)} & & \text { whenever } \xi \geq c, \\
f\left(\xi^{\nu}\right) & \leq c \nu f(\xi) & & \text { whenever } \xi \geq c \text { and } \nu \geq 1 .
\end{aligned}
$$

Choose $d=4 c^{3}$, and let $r \in \mathbb{R}_{+}$be such that $f(r)>d^{2}$. In order to verify that this choice works let $x \in S$ with $\|x\|_{r} \neq\|x\|_{\infty}$. Let $l \geq r$ and $E_{1}<\ldots<E_{l}$ be such that

$$
\|x\|_{r}=\frac{1}{f(l)} \sum_{i=1}^{l}\left\|E_{i}(x)\right\| .
$$

For $\widetilde{r}, \widetilde{R} \in \mathbb{R}$ with $2 \leq \widetilde{r}<\widetilde{R}$, let $M=M(\widetilde{r}, \widetilde{R}):=\left\{i \leq l: \operatorname{char}\left(E_{i}(x)\right) \in\right.$ $[\widetilde{r}, \widetilde{R})\}$, and for $i \in M$ let $l_{i} \in[\widetilde{r}, \widetilde{R})$ be the character of $E_{i}(x)$. We choose for each $i \in M$ finite subsets $E_{1}^{i}<\ldots<E_{l_{i}}^{i}$ of $E_{i}$ so that

$$
\left\|E_{i}(x)\right\|=\frac{1}{f\left(l_{i}\right)} \sum_{j=1}^{l_{i}}\left\|E_{j}^{i}(x)\right\| .
$$

Now we observe that the set $\left\{E_{i}: i \notin M\right\} \cup \bigcup_{i \in M}\left\{E_{j}^{i}: 1 \leq j \leq l_{i}\right\}$ is well ordered by $<$ and its cardinality is $l-\# M+\sum_{i \in M} l_{i}$, which is at least $l$ and at most $l \widetilde{R}$. Thus we deduce:

$$
\begin{aligned}
\|x\|_{r} & =\frac{1}{f(l)} \sum_{i=1}^{l}\left\|E_{i}(x)\right\| \\
& \geq \frac{1}{f\left(l-\# M+\sum_{i \in M} l_{i}\right)}\left[\sum_{i=1, i \notin M}^{l}\left\|E_{i}(x)\right\|+\sum_{i \in M} \sum_{j=1}^{l_{i}}\left\|E_{j}^{i}(x)\right\|\right] \\
& \geq \frac{1}{f(l \widetilde{R})}\left[\sum_{i=1, i \notin M}^{l}\left\|E_{i}(x)\right\|+\sum_{i \in M} f\left(l_{i}\right)\left\|E_{i}(x)\right\|_{l_{i}}\right]
\end{aligned}
$$




$$
\begin{aligned}
& \geq \frac{1}{f(l \widetilde{R})}\left[\sum_{i=1}^{l}\left\|E_{i}(x)\right\|+\sum_{i \in M}(f(\widetilde{r})-1)\left\|E_{i}(x)\right\|\right] \\
& \left.\geq \frac{1}{f(l \widetilde{R})}\left[\sum_{i=1}^{l}\left\|E_{i}(x)\right\|+\frac{1}{c} f(\widetilde{r}) \sum_{i \in M}\left\|E_{i}(x)\right\|\right] \quad \text { (by }(4)\right) .
\end{aligned}
$$

Solving for $(1 / f(l)) \sum_{i \in M}\left\|E_{i}(x)\right\|$ leads to the inequalities

$$
\begin{aligned}
\frac{1}{f(l)} \sum_{i \in M}\left\|E_{i}(x)\right\| & \leq \frac{1}{f(l)}\left[\frac{1}{f(l)}-\frac{1}{f(l \widetilde{R})}\right] \frac{c f(l \widetilde{R})}{f(\widetilde{r})} \sum_{i=1}^{l}\left\|E_{i}(x)\right\| \\
& =\frac{c}{f(\widetilde{r})} \frac{f(l \widetilde{R})-f(l)}{f(l)}\|x\|_{r} \\
& \left.\leq c^{2} \frac{f(\widetilde{R})}{f(\widetilde{r}) f(l)}\|x\|_{r} \leq c^{2} \frac{f(\widetilde{R})}{f(\widetilde{r}) f(r)}\|x\|_{r} \quad \text { (by } 5\right) .
\end{aligned}
$$

Choosing for the pair $(\widetilde{r}, \widetilde{R})$ the values $\left(2, r^{1 / \sqrt{f(r)}}\right),\left(r^{1 / \sqrt{f(r)}}, r\right)$, $\left(r, r^{\sqrt{f(r)}}\right)$, and $\left(r^{\sqrt{f(r)}}, r^{f(r)}\right)$ we deduce from the inequalities $(6)$ and $(7)$ in each case that

$$
\frac{f(\widetilde{R})}{f(\widetilde{r}) f(r)} \leq \frac{c}{\sqrt{f(r)}},
$$

which together with (9) implies that

$$
\frac{1}{f(l)} \sum_{2 \leq \operatorname{char}\left(E_{i}(x)\right)<r f(r)}\left\|E_{i}(x)\right\| \leq \frac{4 c^{3}}{\sqrt{f(r)}}\|x\|_{r}
$$

and, thus, that

yielding the lemma.

$$
\|x\|_{r} \leq \frac{d}{\sqrt{f(r)}}\|x\|_{r}+\frac{1}{f(l)} \sum_{i=1}^{l}\left\|E_{i}(x)\right\|_{r f(r)},
$$

REMARK. Note that in the proof of Lemma 2.6 the only properties of the function $f$ which were needed were that it is increasing and that there is a $c>2$ so that the inequalities (4)-(7) hold. Thus if $l_{0} \in \mathbb{N}$ and $g$ : $\left[l_{0}, \infty\right) \rightarrow(1, \infty)$ is an increasing function so that there is a $c>l_{0}$ for which (4) (whenever $\xi \geq l_{0}$ ), (5), (6) and (7) hold then the conclusion of Lemma 2.6 holds for the completion of $c_{00}$ under the norm $\langle\langle\cdot\rangle\rangle$ defined implicitly by

$$
\langle\langle x\rangle\rangle=\|x\|_{\ell_{\infty}} \vee \sup _{l \geq l_{0}, E_{1}<\ldots<E_{l}} \frac{1}{g(l)} \sum_{i=1}^{l}\left\langle\left\langle E_{i}(x)\right\rangle\right\rangle \quad \text { whenever } x \in c_{00} .
$$

Proof of Theorem 2.4. Let $\left(y_{n}\right),\left(k_{n}\right)$ and $\left(\varepsilon_{n}\right)$ be as in the statement of Theorem 2.4 and let $d \geq 1$ be as in Lemma 2.6. For $r \geq 1$ we put $r_{0}:=r$ and, assuming $r_{k}$ was already defined, we let $r_{k+1}=r_{k}^{f\left(r_{k}\right)}$. From the properties 
of the function $f$ it follows that there is an $R>1$ so that the value

$$
\beta(r):=\prod_{k=0}^{\infty}\left(\frac{1}{1-d / \sqrt{f\left(r_{k}\right)}}\right) \frac{f\left(9 r_{k}\right)}{f\left(r_{k}\right)}
$$

is finite whenever $r \geq R$. By induction we will show that for every $m \in \mathbb{N}$ and every $\left(\alpha_{i}\right)_{i=1}^{m} \subset \mathbb{R}$,

$$
\left\|\left|\sum_{i=1}^{m} \alpha_{i} y_{i}\right|\right\|_{r} \leq \beta(r) \max _{i_{0} \geq 1}\left[\left|\alpha_{i_{0}}\right|+\left\|\sum_{i>i_{0}} \alpha_{i} e_{i}\right\|+\sum_{i=1}^{m}\left|\alpha_{i}\right| \varepsilon_{i}\right] .
$$

Since $\|\cdot\|_{r}$ is equivalent to $\|\cdot\|$ for all $r \geq 1$, since $\sum \varepsilon_{i}<\infty$, and since $\left\|\sum \alpha_{i} y_{i}\right\| \geq\left\|\sum \alpha_{i} e_{i}\right\| \geq \max _{i \in \mathbb{N}}\left|\alpha_{i}\right|$ for $\left(\alpha_{i}\right) \in c_{00}$ this will prove the assertion of Theorem 2.4.

For $m=1$ the claim is trivial. Assume it is true for all positive integers smaller than some $m>1$ and let $r \geq R$ and $\left(\alpha_{i}\right)_{i=1}^{m} \in c_{00}$. Let $y=\sum_{i=1}^{m} \alpha_{i} y_{i}$. If $\|y\|_{r}=\|y\|_{\ell_{\infty}}$ the assertion follows easily since $\|y\|_{\ell_{\infty}} \leq \max _{i \leq m}\left|\alpha_{i}\right|$. Otherwise we can use Lemma 2.6 in order to find an $l \geq r$ and finite subsets $E_{1}<\ldots<E_{l}$ of $\mathbb{N}$ so that $($ with $\gamma(r)=1 /(1-d / \sqrt{f(r)})$ )

$$
\|y\|_{r} \leq \gamma(r) \frac{1}{f(l)} \sum_{j=1}^{l}\left\|E_{j}(y)\right\|_{r f(r)} .
$$

We can assume that for all $j \leq l, E_{j} \subset \bigcup_{i=1}^{m} \operatorname{supp}\left(y_{i}\right)$. For $j=1, \ldots, l$ we put $E_{j}^{1}:=E_{j} \cap \operatorname{supp}\left(y_{s(j)}\right), E_{j}^{2}:=E_{j} \cap \operatorname{supp}\left(y_{t(j)}\right)$ and $E_{j}^{3}=E_{j} \backslash\left(E_{j}^{1} \cup E_{j}^{2}\right)$ where $s(j):=\min \left\{i: E_{j} \cap \operatorname{supp}\left(y_{i}\right) \neq \emptyset\right\}$ and $t(j):=\max \left\{i: E_{j} \cap \operatorname{supp}\left(y_{i}\right)\right.$ $\neq \emptyset\}$. We put $\widetilde{\mathcal{E}}:=\left\{E_{j}^{1}, E_{j}^{2}, E_{j}^{3}: j \leq l\right\} \backslash\{\emptyset\}$ and note that $\widetilde{\mathcal{E}}$ can be ordered into $\widetilde{\mathcal{E}}=\left\{\widetilde{E}_{1}, \ldots, \widetilde{E}_{\tilde{l}}\right\}$ with $l \leq \tilde{l} \leq 3 l$ and $\widetilde{E}_{1}<\ldots<\widetilde{E}_{\tilde{l}}$.

Secondly, we observe that $\widetilde{\mathcal{E}}$ can be partitioned into $m+1$ sets $\widetilde{\mathcal{E}}_{0}, \widetilde{\mathcal{E}}_{1}, \ldots$ $\ldots, \widetilde{\mathcal{E}}_{m}$ defined in the following way: $\widetilde{\mathcal{E}}_{0}:=\left\{E \in \widetilde{\mathcal{E}}: E\right.$ fits with $\left.\left(\operatorname{supp}\left(y_{i}\right)\right)_{i \in \mathbb{N}}\right\}$ (where we say that $E$ fits with a sequence $\left(A_{n}\right)$ of disjoint subsets of $\mathbb{N}$ if for all $n, E \cap A_{n} \neq \emptyset$ implies that $A_{n} \subset E$ ) and for $1 \leq i \leq m$ we let $\widetilde{\mathcal{E}}_{i}:=\left\{E: E \in \widetilde{\mathcal{E}}\right.$ and $\left.E \subsetneq \operatorname{supp}\left(y_{i}\right)\right\}$.

For $i=1, \ldots, m$ we let $l_{i}:=\# \widetilde{\mathcal{E}}_{i}$ (note that $\widetilde{\mathcal{E}}_{i}$ may be empty) and let $i_{0}=1$ if $l_{i} \leq k_{i-1}$ for all $i \leq m$, otherwise put $i_{0}:=\max \left\{i \leq m: l_{i}>k_{i-1}\right\}$.

From (12) we now deduce that (recall that $r_{1}=r^{f(r)}$ )

$$
\begin{aligned}
& \|y\|_{r} \leq \frac{\gamma(r)}{f(l)} \sum_{j=1}^{\widetilde{l}}\left\|\widetilde{E}_{j}(y)\right\|_{r_{1}} \\
& \leq \frac{\gamma(r)}{f(l)} \sum_{j=1}^{\widetilde{l}}\left[\|\| \widetilde{E}_{j}\left(\sum_{i<i_{0}} \alpha_{i} y_{i}\right)\|\|_{r_{1}}+\left\|\widetilde{E}_{j}\left(\alpha_{i_{0}} y_{i_{0}}\right)\right\|_{r_{1}}+\left\|\widetilde{E}_{j}\left(\sum_{i>i_{0}} \alpha_{i} y_{i}\right)\right\| \|_{r_{1}}\right]
\end{aligned}
$$




$$
\begin{aligned}
& \leq \frac{\gamma(r)}{f(l)} \sum_{i<i_{0}} \sum_{j=1}^{\widetilde{l}}\left|\alpha_{i}\right| \cdot\left\|E_{j}\left(y_{i}\right)\right\|+\frac{\gamma(r) f(\widetilde{l})}{f(l)} \frac{\left|\alpha_{i_{0}}\right|}{f(\widetilde{l})} \sum_{j=1}^{\widetilde{l}}\left\|\widetilde{E}_{j}\left(y_{i_{0}}\right)\right\| \\
& \quad+\frac{\gamma(r)}{f(l)} \sum_{i>i_{0}, \mathcal{E}_{i} \neq \emptyset}\left|\alpha_{i}\right| \sum_{E \in \mathcal{E}_{i}}\left\|E\left(y_{i}\right)\right\|+\frac{\gamma(r)}{f(l)} \sum_{E \in \mathcal{E}_{0}}\left\|E\left(\sum_{i>i_{0}} \alpha_{i} y_{i}\right)\right\| \|_{r_{1}} .
\end{aligned}
$$

If $i_{0} \neq 1$ we deduce that the first term in the above sum can be estimated as follows (we use condition (3) of the statement of Theorem 2.4 and note that from the choice of $i_{0}$ it follows that $l \geq \widetilde{l} / 3 \geq l_{i_{0}} / 3 \geq k_{i_{0}-1} / 3 \geq k_{i} / 3$ for $\left.i<i_{0}\right)$ :

$$
\frac{\gamma(r)}{f(l)} \sum_{i<i_{0}} \sum_{j=1}^{\tilde{l}}\left|\alpha_{i}\right| \cdot\left\|E_{j}\left(y_{i}\right)\right\| \leq \gamma(r) \sum_{i<i_{0}} \frac{\left|\alpha_{i}\right|}{f\left(k_{i} / 3\right)} \cdot \# \operatorname{supp}\left(y_{i}\right) \leq \gamma(r) \sum_{i=1}^{i_{0}-1} \varepsilon_{i}\left|\alpha_{i}\right| .
$$

The second term can be estimated as follows:

$$
\frac{\gamma(r) f(\widetilde{l})}{f(l)}\left|\alpha_{i_{0}}\right| \frac{1}{f(\widetilde{l})} \sum_{j=1}^{\widetilde{l}}\left\|\widetilde{E}_{j}\left(y_{i_{0}}\right)\right\| \leq \frac{\gamma(r) f(3 l)}{f(l)}\left|\alpha_{i_{0}}\right| \cdot\left\|y_{i_{0}}\right\| \leq \frac{\gamma(r) f(3 r)}{f(r)}\left|\alpha_{i_{0}}\right| .
$$

By condition (2) of the statement of Theorem 2.4 and the definition of $i_{0}$ we deduce that $\sum_{E \in \mathcal{E}_{i}}\left\|E\left(y_{i}\right)\right\| \leq 1+\varepsilon_{i}$ if $i>i_{0}$ and $\mathcal{E}_{i} \neq \emptyset$. Thus, we observe for the third term that

$$
\frac{\gamma(r)}{f(l)} \sum_{i>i_{0}, \mathcal{E}_{i} \neq \emptyset}\left|\alpha_{i}\right| \sum_{E \in \mathcal{E}_{i}}\left\|E\left(y_{i}\right)\right\| \leq \frac{\gamma(r)}{f(l)} \sum_{i>i_{0}, \mathcal{E}_{i} \neq \emptyset}\left(1+\varepsilon_{i}\right)\left|\alpha_{i}\right| .
$$

For the last term we apply the induction hypothesis and find for each $E \in \mathcal{E}_{0}$ an $i_{E} \in\left\{i>i_{0}: \operatorname{supp}\left(y_{i}\right) \subset E\right\} \cup\{0\}$ so that

$$
\begin{aligned}
& \sum_{E \in \mathcal{E}_{0}}\|\| E\left(\sum_{i>i_{0}} \alpha_{i} y_{i}\right)\|\|_{r_{1}} \\
& \quad \leq \beta\left(r_{1}\right) \sum_{E \in \mathcal{E}_{0}}\left[\left|\alpha_{i_{E}}\right|+\left\|\sum_{\substack{i>i_{E} \\
\operatorname{supp}\left(y_{i}\right) \subset E}} \alpha_{i} e_{i}\right\|+\sum_{\operatorname{supp}\left(y_{i}\right) \subset E}\left|\alpha_{i}\right| \varepsilon_{i}\right] .
\end{aligned}
$$

Let $\mathcal{A}=\left\{\{i\}: i>i_{0}, \mathcal{E}_{i} \neq \emptyset\right\} \cup\left\{\left\{i_{E}\right\}: E \in \mathcal{E}_{0}\right\} \cup\left\{\left\{i>i_{E}: \operatorname{supp}\left(y_{i}\right) \subseteq E\right\}\right.$ : $\left.E \in \mathcal{E}_{0}\right\} \backslash\{\emptyset\}$ and note that $\mathcal{A}$ consists of subsets of $\left\{i_{0}+1, i_{0}+2, \ldots\right\}$, has at most $3 \widetilde{l} \leq 9 l$ elements and is well ordered by $<$. Finally we deduce from (13) and the above estimates that

$$
\begin{aligned}
\|y\|_{r} \leq & \gamma(r) \sum_{i<i_{0}}\left|\alpha_{i}\right| \varepsilon_{i}+\frac{\gamma(r) f(3 r)}{f(r)}\left|\alpha_{i_{0}}\right|+\frac{\gamma(r)}{f(l)} \sum_{i>i_{0}, \mathcal{E}_{i} \neq \emptyset}^{m}\left|\alpha_{i}\right| \\
& +\frac{\gamma(r)}{f(l)} \sum_{i=1, \mathcal{E}_{i} \neq \emptyset}^{m} \varepsilon_{i}\left|\alpha_{i}\right|+\frac{\gamma(r) \beta\left(r_{1}\right)}{f(l)} \sum_{E \in \mathcal{E}_{0}}\left|\alpha_{i_{E}}\right|
\end{aligned}
$$




$$
\begin{aligned}
& +\frac{\gamma(r) \beta\left(r_{1}\right)}{f(l)} \sum_{E \in \mathcal{E}_{0}}\left\|\sum_{i>i_{E}, \operatorname{supp}\left(y_{i}\right) \subset E} \alpha_{i} e_{i}\right\| \\
& +\frac{\gamma(r) \beta\left(r_{1}\right)}{f(l)} \sum_{\substack{\operatorname{supp}\left(y_{i}\right) \subset E \\
E \in \mathcal{E}_{0}}}\left|\alpha_{i}\right| \varepsilon_{i} \\
& \leq \beta(r)\left[\left|\alpha_{i_{0}}\right|+\frac{f(r)}{f(l) f(9 r)} \sum_{A \in \mathcal{A}}\left\|A\left(\sum_{i=1}^{m} \alpha_{i} e_{i}\right)\right\|+\sum_{i=1}^{m} \varepsilon_{i}\left|\alpha_{i}\right|\right] \\
& \leq \beta(r)\left[\left|\alpha_{i_{0}}\right|+\frac{f(r)}{f(9 r)} \frac{f(9 l)}{f(l)}\left\|\sum_{i=1}^{m} \alpha_{i} e_{i}\right\|+\sum_{i=i_{0}+1}^{m} \varepsilon_{i}\left|\alpha_{i}\right|\right] \quad(\text { since } \# \mathcal{A} \leq 9 l) \\
& \leq \beta(r)\left[\left|\alpha_{i_{0}}\right|+\left\|\sum_{i=1}^{m} \alpha_{i} e_{i}\right\|+\sum_{i=i_{0}+1}^{m} \varepsilon_{i}\left|\alpha_{i}\right|\right] \quad(\text { since } l \geq r) .
\end{aligned}
$$

This proves the induction step and completes the proof of Theorem 2.4.

3. The Banach space $S$ is subsequentially prime. The main result of this section is the following theorem.

TheOREM 3.1. The space $S$ is subsequentially prime.

Theorem 3.1 will essentially follow from A. Pełczyński's decomposition method and the following theorem.

THEOREM 3.2. Let $\left(x_{i}\right)$ be a normalized block sequence of $\left(e_{i}\right)$ in $S$ and let $\left(k_{i}\right)$ be a subsequence of $\mathbb{N}$. There exists a subsequence $\left(y_{i}\right)$ of $\left(x_{i}\right)$ with the following property: If $\left(s_{i}\right)$ and $\left(t_{i}\right)$ are strictly increasing in $\mathbb{N}$, and $s_{i}, t_{i} \leq k_{i}$ for $i \in \mathbb{N}$, then $\left(y_{s_{i}}\right)$ and $\left(y_{t_{i}}\right)$ are equivalent.

Before giving the proof of Theorem 3.2 we need a result, for which we introduce the following norm $\langle\langle\cdot\rangle\rangle$ on $c_{00}$. For $l \geq 3$ define $g(l)=\log _{2}(1+l / 2)$ and let $\langle\langle\cdot\rangle\rangle$ be the norm which is implicitly defined by

$$
\langle\langle x\rangle\rangle=\|x\|_{\ell_{\infty}} \vee \sup _{l \geq 3, E_{1}<\ldots<E_{l}} \frac{1}{g(l)} \sum_{i=1}^{l}\left\langle\left\langle E_{i}(x)\right\rangle\right\rangle \quad \text { whenever } x \in c_{00} .
$$

LEMMA 3.3. The norm $\langle\langle\cdot\rangle\rangle$ is equivalent to the norm $\|\cdot\|$ on $S$.

Proof. First note that since $f(l) \geq g(l+1)$ whenever $l \geq 2$ it follows that $\langle\langle\cdot\rangle\rangle \geq\|\cdot\|$. Thus, we only have to show that for some constant $C$ it follows that $\langle\langle\cdot\rangle\rangle \leq C\|\cdot\|$. The proof will be similar to that of Theorem 2.4.

For $l, r \in[3, \infty)$ let $\langle\langle\cdot\rangle\rangle_{l}$ and $\left\langle\langle\langle\cdot\rangle\rangle_{r}\right.$ be defined as $\|\cdot\|_{l}$ and $\|\cdot\|_{r}$ respectively. Let $\tilde{d}>1$ be chosen so that the statement of Lemma 2.6 holds (see 
remark after the proof of Lemma 2.6), i.e. so that

$$
\langle\langle\langle x\rangle\rangle\rangle_{r} \leq\left[\frac{1}{1-\widetilde{d} / \sqrt{g(r)}}\right] \sup _{\substack{l \geq r \\ E_{1}<\ldots<E_{l}}} \frac{1}{g(l)} \sum_{i=1}^{l}\left\langle\left\langle\left\langle E_{i}(x)\right\rangle\right\rangle\right\rangle_{r^{g(r)}}
$$

if $r \in \mathbb{R}_{+}$with $g(r)>\widetilde{d^{2}}$, and $x \in c_{00}$ with $\left\langle\left\langle\left\langle x_{r}\right\rangle\right\rangle\right\rangle \neq\|x\|_{\ell_{\infty}}$.

For $r \in[3, \infty)$ we define $r_{0}=r$ and, assuming that $r_{k}$ had been defined, let $r_{k+1}=r_{k}^{g\left(r_{k}\right)}$. Then we deduce that there is an $R \geq 3$ so that for all $r \geq R$

$$
\widetilde{\beta}(r)=\prod_{k=0}^{\infty} \frac{1}{1-\widetilde{d} / \sqrt{g\left(r_{k}\right)}} \frac{g\left(2 r_{k}\right)}{g\left(r_{k}\right)}
$$

is finite. By induction on $m \in \mathbb{N}$ we prove that for each $x \in c_{00}$ so that $\# \operatorname{supp}(x) \leq m$ and $r \geq R$ it follows that

$$
\langle\langle\langle x\rangle\rangle\rangle_{r} \leq \widetilde{\beta}(r)\|x\| .
$$

Assume that (15) is true for all $z \in c_{00}$ for which $\# \operatorname{supp}(z)<m$ and assume that $x \in c_{00}$ with $\# \operatorname{supp}(x)=m$. If $\left\langle\langle\langle x\rangle\rangle_{r}=\|x\|_{\ell_{\infty}}\right.$ the claim follows immediately. Otherwise it follows from (14) that for some $l \geq r$, $l \in \mathbb{N}$, and some choice of $E_{1}<\ldots<E_{l}$ we have

$$
\begin{aligned}
&\left\langle\langle\langle x\rangle\rangle_{r}\right. \leq\left[\frac{1}{1-\widetilde{d} / \sqrt{g(r)}}\right] \frac{1}{g(l)} \sum_{i=1}^{l}\left\langle\left\langle\left\langle E_{i}(x)\right\rangle\right\rangle\right\rangle_{r^{g}}(r) \\
& \leq \widetilde{\beta}\left(r^{g(r)}\right)\left[\frac{1}{1-\widetilde{d} / \sqrt{g(r)}}\right] \frac{1}{g(l)} \sum_{i=1}^{l}\left\|E_{i}(x)\right\| \\
&=\widetilde{\beta}(r) \frac{g(r)}{g(2 r)} \frac{1}{g(l)} \sum_{i=1}^{l}\left\|E_{i}(x)\right\| \leq \widetilde{\beta}(r)\|x\| \\
& \quad\left(\text { since } \frac{g(r)}{g(2 r)} \leq \frac{g(l)}{g(2 l)}=\frac{g(l)}{f(l)}\right),
\end{aligned}
$$

which finishes the induction step and the proof of Lemma 3.3.

Proof of Theorem 3.2. First we note that we can assume that $\lim _{n \rightarrow \infty}\left\|x_{n}\right\|_{\ell_{\infty}}=0$. Indeed, from Theorem 2.4 it follows that there is a normalized block $\left(z_{k}\right)$ in $S$ which is equivalent to the unit vector basis $\left(e_{k}\right)$ and has the property that $\lim _{n \rightarrow \infty}\left\|z_{n}\right\|_{\ell_{\infty}}=0$. Thus, we could replace each $x_{n}$ by the vector $x_{n}^{\prime}$ in the span of $\left(z_{k}\right)$ whose coordinates with respect to the $z_{k}$ 's are the coordinates of $x_{n}$ with respect to the $e_{k}$ 's. 
Let $\varepsilon>0$, and $y \in c_{00}$ with $\|y\|=1$ and $\|y\|_{\infty} \leq \varepsilon / 2$. We can write $y$ as $y=\sum_{i=1}^{l(y, \varepsilon)} y(i, \varepsilon)$, where $l(y, \varepsilon) \in \mathbb{N}$ and $y(1, \varepsilon)<y(2, \varepsilon)<\ldots<y(l(y, \varepsilon), \varepsilon)$ such that $\|y(i, \varepsilon)\| \leq \varepsilon$ for $i=1, \ldots, l(y, \varepsilon)$. Furthermore we could choose the $y(i, \varepsilon)$ 's to have maximal support in the following sense. First we choose $y(i, \varepsilon)=\left[1, n_{1}\right](y)$ with $n_{1} \in \mathbb{N}$ being the largest $n \in \mathbb{N}, n \leq \max \operatorname{supp}(y)$, so that $\|[1, n](y)\| \leq \varepsilon$. Then we choose $n_{2}>n_{1}$ being the largest $n \in \mathbb{N}$, $n \leq \operatorname{supp}(y)$, so that $\left\|\left[n_{1}+1, n_{2}\right](y)\right\| \leq \varepsilon$. We can continue this way until we have exhausted the support of $y$. This defines $l(y, \varepsilon)$ and the vectors $(y(i, \varepsilon))_{i=1}^{l(y, \varepsilon)}$ uniquely and from the assumption that $\|y\|_{\infty} \leq \varepsilon / 2$ it follows that $\varepsilon / 2 \leq\|y(i, \varepsilon)\|$ for all $i \in\{1, \ldots, l(y, \varepsilon)-1\}$. From the definition of the norm of $S$ is follows that

$\frac{\ell(y, \varepsilon)-1}{f(\ell(y, \varepsilon))} \frac{\varepsilon}{2} \leq \frac{1}{f(l(y, \varepsilon))} \sum_{i=1}^{l(y, \varepsilon)}\|y(i, \varepsilon)\| \leq\|y\|=1 \leq \sum_{i=1}^{l(y, \varepsilon)}\|y(i, \varepsilon)\| \leq l(y, \varepsilon) \varepsilon$.

Thus for $\varepsilon>0$ there are two numbers $H(\varepsilon) \geq h(\varepsilon)$ in $\mathbb{N}$ with $h(\varepsilon) \nearrow \infty$ if $\varepsilon \searrow 0$, and so that for any $y \in c_{00}$ with $\|y\|=1$ and $\|y\|_{\infty} \leq \varepsilon / 2$ it follows that $h(\varepsilon) \leq l(y, \varepsilon) \leq H(\varepsilon)$.

We now apply this "splitting procedure" to the elements of our sequence $\left(x_{n}\right)$. By induction on $n \in \mathbb{N}$ we find an infinite subset $M_{n}$ of $\mathbb{N}$ with $\mathbb{N} \supset$ $M_{1} \supset \ldots \supset M_{n}$ and $\min M_{1}<\ldots<\min M_{n}$, and numbers $\varepsilon(n)>0$ and $p(n) \in \mathbb{N}$ satisfying the following three properties:

$$
\text { For all } m \in M_{n} \text { we have } l\left(\varepsilon(n), x_{m}\right)=p(n) \text {. }
$$

For any choice of $n \leq s_{0}<s_{1}<\ldots<s_{k_{n}+1}$ and $n \leq t_{0}<t_{1}<\ldots$ $<t_{k_{n}+1}$ in $M_{n}$ it follows that $\left(x_{s_{j}}(i, \varepsilon(n))\right)_{0 \leq j \leq k_{n}+1, i \leq p(n)} \approx_{1+\varepsilon(n)}$ $\left(x_{t_{j}}(i, \varepsilon(n))\right)_{0 \leq j \leq k_{n}+1, i \leq p(n)}$.

If $n>1$ it follows that $\sum_{1=i}^{n-1}\left\|x_{\min M_{i}}\right\|_{\ell_{1}}<f(h(\varepsilon(n))) 2^{-n}$ and $p(n-1) \varepsilon(n)<2^{-n}$.

For $n=1$ we simply choose $\varepsilon(1)=1$ (thus $\left.l\left(x_{n}, \varepsilon(1)\right)=1\right), p(1)=1$, and using compactness and the usual stabilization argument we can pass to a subsequence $M_{1}$ of $\mathbb{N}$ so that (17) holds. Assuming we made our choices of $M_{j}, \varepsilon(j)$, and $p(j)$ for all $j<n$, we first choose $\varepsilon(n)$ so that (18) is satisfied (recall that $h(\varepsilon) \nearrow \infty$ for $\varepsilon \searrow 0$ ), and then again using compactness and the usual stabilization argument we can pass to an $M_{n} \subset M_{n-1} \backslash\left\{\min M_{n-1}\right\}$ and find a $p(n) \in \mathbb{N}$ so that $\left\|x_{m}\right\| \leq \varepsilon(n) / 2$ whenever $m \in M_{n}$ and so that (16) and (17) are satisfied.

For $n \in \mathbb{N}$ we now define $y_{n}=x_{\min \left(M_{n}\right)}$ and $y_{n}(i, j)=y_{n}(i, \varepsilon(j))$ if $i \leq n$ and $j \leq p(i)$, and prove the following claim by induction on $N \in \mathbb{N}$ :

Claim. For every $n \in \mathbb{N}$, every $K, L \in\{1, \ldots, p(n)\}$, every $\left(\alpha_{i}\right)_{i=0}^{n+N+1}$ $\subset \mathbb{R}$, and every choice of $n \leq s_{0}<s_{1}<\ldots<s_{n+N+1}$ and $n \leq t_{0}<t_{1}<\ldots$ 
$<t_{n+N+1}$ with $s_{i}, t_{i} \leq k_{i+n}$ for $i=0,1 \ldots, n+N+1$, it follows for

$$
\begin{aligned}
& x=\alpha_{0} \sum_{j=K}^{p(n)} y_{s_{0}}(n, j)+\sum_{i=1}^{N} \alpha_{i} y_{s_{i}}+\alpha_{N+n+1} \sum_{j=1}^{L} y_{s_{n+N+1}}(n, j), \\
& \widetilde{x}=\alpha_{0} \sum_{j=K}^{p(n)} y_{t_{0}}(n, j)+\sum_{i=1}^{N} \alpha_{i} y_{t_{i}}+\alpha_{N+n+1} \sum_{j=1}^{L} y_{t_{n+N+1}}(n, j)
\end{aligned}
$$

that

$$
\|x\| \leq c(n) \max _{E<F}[\langle\langle E(\widetilde{x})\rangle\rangle+\langle\langle F(\widetilde{x})\rangle\rangle]
$$

where

$$
c(n)=\sum_{i=n}^{N} 2^{-i}+\varepsilon(i) .
$$

Since by Lemma 3.3, $\langle\langle\cdot\rangle\rangle$ is an equivalent norm on $S$ (and therefore also the norm $c_{00} \ni x \mapsto \max _{E<F}\langle\langle E(x)\rangle\rangle+\langle\langle F(x)\rangle\rangle$ has this property) the claim implies the theorem.

For $N=0$ the claim follows directly from (17). Assume the claim to be true for $\widetilde{N}<N$ and let $x$ and $\widetilde{x}$ be given as in (19) and (20).

We choose $l \in \mathbb{N}$ so that $\|x\|=\|x\|_{l}$. If $l<p\left(k_{n}\right)$ we let $i_{0}=0$, and otherwise we choose $i_{0} \in \mathbb{N} \cup\{0\}$ so that $p\left(k_{i_{0}+n}\right) \leq l<p\left(k_{i_{0}+n+1}\right)$. We split $x$ into three vectors $x^{(1)}, x^{(2)}$ and $x^{(3)}$ as follows. If $i_{0}=0$ we let $x^{(1)}=0$, otherwise we put

$$
x^{(1)}=\alpha_{0} \sum_{j=K}^{p(n)} y_{s_{0}}(n, j)+\sum_{i=1}^{\left(i_{0}-1\right) \wedge N} \alpha_{i} y_{s_{i}},
$$

and $\widetilde{x}^{(1)}$ is defined as $x^{(1)}$, by replacing the $s_{i}$ 's by $t_{i}$ 's. From (18) and the choice of $i_{0}$ it follows that (note that $h\left(\varepsilon\left(s_{i_{0}-1}+1\right)\right) \leq h\left(\varepsilon\left(s_{i_{0}}\right)\right) \leq p\left(s_{i_{0}}\right) \leq$ $\left.p\left(k_{n+i_{0}}\right) \leq l\right)$

$$
\begin{aligned}
\left\|x^{(1)}\right\|_{l} & \leq \max _{0 \leq i \leq n+N}\left|\alpha_{i}\right| \frac{1}{f(l)} \sum_{i=0}^{\left(i_{0}-1\right) \wedge N}\left\|y_{s_{i}}\right\|_{l_{1}} \leq\|\widetilde{x}\| \frac{f\left(h\left(\varepsilon\left(s_{i_{0}}\right)\right)\right)}{f(l)} \\
& \leq\|\widetilde{x}\| 2^{-n-i_{0}} .
\end{aligned}
$$

Secondly, if we let

$$
x^{(2)}= \begin{cases}\alpha_{0} \sum_{j=K}^{p(n)} y_{s_{0}}(n, j)+\sum_{i=1}^{\left(k_{n}+1\right) \wedge N} \alpha_{i} y_{s_{i}} & \text { if } i_{0}=0, \\ \sum_{i=\left(i_{0}-1\right) \wedge N+1} \alpha_{\left.n+i_{0}+1\right) \wedge N} y_{s_{i}} & \text { if } i_{0} \neq 0,\end{cases}
$$


and $\widetilde{x}^{(1)}$ is defined as $x^{(1)}$, by replacing the $s_{i}$ 's by $t_{i}$ 's, we deduce from (17) that

$$
\left\|x^{(2)}\right\| \leq\left(1+\varepsilon\left(s_{i_{0}}\right)\right)\left\|\widetilde{x}^{(2)}\right\| \leq\left(1+\varepsilon\left(n+i_{0}\right)\right)\left\|\widetilde{x}^{(2)}\right\| .
$$

Finally we let

$$
x^{(3)}=x-x^{(1)}-x^{(2)}=\sum_{i=k_{n+i_{0}}+2}^{N} \alpha_{i} y_{s_{i}}+\alpha_{N+n+1} \sum_{j=L}^{p(n)} y_{s_{n+N+1}}(n, j)
$$

and, again, define $\widetilde{x}^{(3)}$ as $x^{(3)}$, by replacing the $s_{i}$ 's by $t_{i}$ 's. Choose $E_{1}<$ $\ldots<E_{l}$ so that

$$
\left\|x^{(3)}\right\|_{l}=\frac{1}{f(l)} \sum_{j=1}^{l}\left\|E_{j}\left(x^{(3)}\right)\right\| .
$$

Note that for any $i \geq k_{n+i_{0}}+2$ it follows that $s_{i} \geq k_{n+i_{0}}+2+n \geq k_{n+i_{0}}+2$ and by (18) it follows that $l \varepsilon\left(k_{n+i_{0}}+2\right) \leq p\left(k_{n+i_{0}}+1\right) \varepsilon\left(k_{n+i_{0}}+2\right)<$ $2^{-k_{n+i_{0}}-1} \leq 2^{-n-i_{0}-1}$. Let $n^{\prime}=k_{n+i_{0}}+2+n$. For each $j=1, \ldots, l$ we can perturb $E_{j}$ into a set $F_{j}$ (by possibly taking some part of the support of some $y_{s_{i}}\left(n^{\prime}, u\right)$ away at the beginning of $E_{j}$ and adding some part of the support of some $y_{s_{j}}\left(n^{\prime}, v\right)$ at the end of $\left.E_{j}\right)$ so that for each $j=1, \ldots, l$, each $i \geq k_{n+i_{0}}+2$, and each $u=1, \ldots, p\left(n^{\prime}\right)$, the set $F_{j}$ either contains $\operatorname{supp}\left(y_{s_{i}}\left(n^{\prime}, u\right)\right)$, or is disjoint from it, and so that

$$
\begin{aligned}
\left\|x^{(3)}\right\|_{l} & =\frac{1}{f(l)} \sum_{j=1}^{l}\left\|F_{j}\left(x^{(3)}\right)\right\|+l \varepsilon\left(n^{\prime}\right) \max _{0 \leq i \leq N+n+1}\left|\alpha_{i}\right| \\
& \leq \frac{1}{f(l)} \sum_{j=1}^{l}\left\|F_{j}\left(x^{(3)}\right)\right\|+2^{-n-i_{0}-1}\|x\| .
\end{aligned}
$$

To each of the $F_{j}\left(x^{(3)}\right)$ we can apply the induction hypothesis and obtain a splitting of each

$$
\widetilde{F}_{j}=\bigcup\left\{\operatorname{supp}\left(y_{t_{i}}\left(n^{\prime}, u\right)\right): i \geq i_{0}+3, u \leq p\left(n^{\prime}\right) \text { and } \operatorname{supp}\left(y_{s_{i}}\left(n^{\prime}, u\right)\right) \subset F_{j}\right\}
$$

$\left(\widetilde{F}_{j}\right.$ is the " $\widetilde{x}^{(3)}$ version of $\left.x^{(3) "}\right)$ into $\widetilde{G}_{j}$ and $\widetilde{H}_{j}$, with $\widetilde{G}_{j}<\widetilde{H}_{j}$ so that

$$
\begin{aligned}
\left\|x^{(3)}\right\|_{l} & \leq 2^{-n-i_{0}-1}\|x\|+\frac{1}{f(l)} c\left(n^{\prime}\right) \sum_{j=1}^{l}\left(\left\langle\left\langle\widetilde{G}_{j}\left(\widetilde{x}^{(3)}\right)\right\rangle\right\rangle+\left\langle\left\langle\widetilde{H}_{j}\left(\widetilde{x}^{(3)}\right)\right\rangle\right\rangle\right) \\
& \leq 2^{-n-i_{0}-1}\|x\|+c\left(n^{\prime}\right)\left\langle\left\langle\widetilde{x}^{(3)}\right\rangle\right\rangle
\end{aligned}
$$

(for the last inequality recall the definition of $\langle\langle\cdot\rangle\rangle$ and that $g(2 l)=f(l)$ ).

Finally, putting (24), (26) and (29) together, observing that $\|\cdot\| \leq\langle\langle\cdot\rangle\rangle$, and that $c(n) \geq 2^{-n-i_{0}}+2^{-n-i_{0}-1}+\varepsilon(n+1)+c\left(n^{\prime}\right)$, we obtain

$$
\begin{aligned}
\|x\|=\|x\|_{l} & \leq\left\|x^{(1)}\right\|_{l}+\left\|x^{(2)}\right\|+\left\|x^{(3)}\right\|_{l} \\
& \leq\|\widetilde{x}\| 2^{-n-i_{0}}+\left(1+\varepsilon\left(n+i_{0}\right)\right)\left\|\widetilde{x}^{(2)}\right\|
\end{aligned}
$$




$$
\begin{aligned}
& +2^{-n-i_{0}-1}\|\widetilde{x}\|+c\left(n^{\prime}\right)\left\langle\left\langle\widetilde{x}^{(3)}\right\rangle\right\rangle+c\left(n^{\prime}\right) \sup _{E<F}(\langle\langle E(\widetilde{x})\rangle\rangle+\langle\langle F(\widetilde{x})\rangle\rangle) \\
& +\left[2^{-n-i_{0}}+2^{-n-i_{0}-1}+\varepsilon(n+1)\right]\|\widetilde{x}\| \\
\leq & c(n) \sup _{E<F}(\langle\langle E(\widetilde{x})\rangle\rangle+\langle\langle F(\widetilde{x})\rangle\rangle),
\end{aligned}
$$

which finishes the induction step and the proof of the theorem.

Proof of Theorem 3.1. Let $\left(x_{n}\right)$ be a seminormalized basic sequence in $S$ whose closed linear span is complemented in $S$. Since $S$ is reflexive we can assume, by passing to an appropriate subsequence, that for some $a \in S$, $x_{n}=a+z_{n}$ where $z_{n}$ is weakly null. Since $a$ is an element of the closed linear span of $\left(x_{n}\right)$ and $\left(x_{n}\right)$ is a seminormalized basic sequence it follows that $a=0$, and thus $\left(x_{n}\right)$ is seminormalized and weakly null. By applying the usual perturbation argument we can assume that $\left(x_{n}\right)$ is a seminormalized block sequence and therefore apply Theorem 3.2 with $k_{n}=2 n+1$ for $n \in \mathbb{N}$ to obtain a subsequence $\left(y_{n}\right)$ so that $\left(y_{n}\right),\left(y_{2 n+1}\right)$ and $\left(y_{2 n}\right)$ are equivalent. In particular it follows for $Y=\left[y_{n}: n \in \mathbb{N}\right]$ that $Y \approx Y \oplus Y$ (the complemented sum of $Y$ with itself). Since $S$ has a subsymmetric basis, it also follows that $S \approx S \oplus S$. This means that we are in a position to apply Pełczyński's decomposition method [13], which is so elegant that we cannot restrain ourselves from repeating it here. We write $S=U \oplus Y$ (note that with $\left[x_{i}: i \in \mathbb{N}\right]$ also $Y$ is complemented in $S$ ) and $Y=V \oplus S$. Then it follows that

$$
S \approx U \oplus Y \approx U \oplus Y \oplus Y \approx S \oplus Y \approx S \oplus V \oplus S \approx S \oplus V \approx Y
$$

\section{References}

[1] C. Bessaga and A. Pełczyński, On subspaces of a space with an absolute basis, Bull. Acad. Polon. Sci. 6 (1958), 313-314.

[2] P. G. Casazza, The Schroeder-Bernstein property for Banach spaces, in: Contemp. Math. 85, Amer. Math. Soc., 1989, 61-77.

[3] P. G. Casazza, W. B. Johnson and L. Tzafriri, On Tsirelson's space, Israel J. Math. 17 (1984), 191-218.

[4] P. G. Casazza and E. Odell, Tsirelson's space and minimal subspaces, in: Texas Functional Analysis Seminar 1982-1983, Longhorn Notes, University of Texas, 1983, 61-72.

[5] P. G. Casazza and T. J. Shura, Tsirelson's Space, Lecture Notes in Math. 1363, Springer, 1989.

[6] T. Figiel and W. B. Johnson, A uniformly convex Banach space which contains no $\ell_{p}$, Compositio Math. 29 (1974), 179-190.

[7] W. T. Gowers, A solution to the Schroeder-Bernstein problem for Banach spaces, Bull. London Math. Soc. 28 (1996), 297-304.

[8] W. T. Gowers and B. Maurey, The unconditional basic sequence problem, J. Amer. Math. Soc. 6 (1993), 851-874. 
[9] W. T. Gowers and B. Maurey, Banach spaces with small spaces of operators, Math. Ann. 307 (1997), 543-568.

[10] D. Kutzarova and P. K. Lin, Remarks about Schlumprecht space, Proc. Amer. Math. Soc. 128 (2000), 2059-2068.

[11] J. Lindenstrauss, On complemented subspaces of $m$, Israel J. Math. 5 (1967), 153156.

[12] J. Lindenstrauss and L. Tzafriri, Classical Banach Spaces I. Sequence Spaces, Springer, 1977.

[13] A. Pełczyński, Projections in certain Banach spaces, Studia Math. 19 (1960), 209228.

[14] T. Schlumprecht, An arbitrarily distortable Banach space, Israel J. Math. 76 (1991), 81-95.

[15] - A complementably minimal Banach space not containing $c_{0}$ or $\ell_{p}$, Seminar Notes in Functional Analysis and Partial Differential Equations, Baton Rouge, LA, 1992.

[16] B. S. Tsirelson, Not every Banach space contains an embedding of $\ell_{p}$ or $c_{0}$, Functional Anal. Appl. 8 (1974), 138-141.

Department of Mathematics

University of South Carolina

Columbia, SC 29208, U.S.A.

E-mail: giorgis@math.sc.edu
Department of Mathematics Texas A\&M University College Station, TX 77843, U.S.A.

E-mail: schlump@math.tamu.edu

Received January 2, 2002

Revised version December 20, 2002 